\begin{tabular}{|c|l|}
\hline Title & Uncertainty quantification of neutron multiplication factors of light water reactor fuels during depletion \\
\hline Author(s) & Chiba, Go; Okumura, Shintaro \\
\hline Citation & $\begin{array}{l}\text { Journal of nuclear science and technology, 55(9), 1043-1053 } \\
\text { https://doi.org/10.1080/00223131.2018.1467803 }\end{array}$ \\
\hline Issue Date & 2018 05-29 \\
\hline Doc URL & http://hdl.handle.net/2115/74434 \\
\hline Rights & This is an A ccepted Manuscript of an article published by Taylor \& Francis in Journal of Nuclear Science and \\
\hline Type & article (author version) \\
\hline File Information & paper.pdf \\
\hline
\end{tabular}

Instructions for use 


\title{
Uncertainty quantification of neutron multiplication factors of light water reactor fuels during depletion
}

\author{
Go Chiba $^{*}$, Shintaro Okumura ${ }^{1}$ \\ ${ }^{1}$ Hokkaido University, Kita-ku, Sapporo 060-8628, Japan
}

Nuclear data-induced uncertainties of infinite neutron multiplication factors $\left(k_{\infty}\right)$ during fuel depletion are quantified in a single cell and a $3 \times 3$ multi-cell including burnable absorbers. Uncertainties of reaction cross sections, fission yields, decay half-lives and decay branching ratios provided in the JENDL libraries are taken into account. $100 \%$ uncertainties are assumed to nuclear data to which uncertainty information are not provided in JENDL. Uncertainties propagation calculations are carried out with the adjoint-based procedure, and required sensitivity profiles of $k_{\infty}$ with respect to these nuclear data are efficiently calculated by the depletion perturbation theory. Covariance matrices for fission yields and decay data in a simplified burnup chain are successfully generated by the stochastic-based procedure. $k_{\infty}$ uncertainties of about $0.6 \%$ during fuel depletion are obtained, and it is shown that actinoids reaction cross sections are dominant contributors. Nuclide-wise decomposition of the uncertainties and observation of component-wise sensitivity profiles provide physical interpretations.

By virtue of the adjoint-based procedure, several parametric surveys are also conducted. Contributions of uncertainties in fission products (FP) nuclides are quantified, and important nuclides and energy ranges are identified for further evaluation of nuclear data of FP nuclides. Effect of cooling period on $k_{\infty}$ uncertainties is also discussed. 
Keywords: nuclear fuel depletion; depletion perturbation theory; uncertainty quatification 


\section{Introduction}

Reliable and proper management of spent nuclear fuels is an important issue, and there are various aspects of it. One of the most important aspects is criticality safety; systems consisting of spent nuclear fuels should be kept a sub-critical state in every probable conditions and situations. Spent nuclear fuels generally contain fission product (FP) nuclides, which are neutron absorbers, and less amount of fissile nuclides than fresh fuels, so reactivity of spent nuclear fuels should be much lower than that of fresh fuels. Thus criticality management of spent nuclear fuels can be attained efficiently if ones can properly consider this reactivity degradation. This concept is referred to as the burnup credit, and has been introduced to criticality management in several countries.

In order to predict reactivity of spent nuclear fuels, ones generally solve the eigenvalue equation describing neutron transport by computer codes, and the calculated eigenvalue corresponds to reactivity. Various kinds of macroscopic neutron reaction cross sections are introduced to the neutron transport equation, so ones have to know accurate nuclear data (microscopic cross sections) in order to accurately predict reactivity. This dependence of reactivity on nuclear data is generally called as static dependence. On the other hand, nuclides number densities are also important when ones consider systems consisting of spent nuclear fuels. Nuclides number densities of spent nuclear fuels are also predicted by computer codes, and nuclear data are used in these calculations. Thus, the calculated nuclides number densities depend on nuclear data, so reactivity of systems consisting of spent nuclear fuels depends indirectly on nuclear data via nuclides number densities. This dependence is called as depletion dependence. These clearly show that nuclear data are one of the most important parameters and quantities in criticality management of spent nuclear fuels.

To properly consider safety margin of design parameters related to criticality safety, uncertainty quantification of predicted reactivity is quite an important subject. Since 
nuclear data are evaluated from results of direct or indirect measurements and model calculations, these should have a certain degree of uncertainty. This nuclear data uncertainty is one of dominant sources in uncertainty of predicted reactivity. Nuclear data themselves and their uncertainty have been evaluated simultaneously, and both of them are stored in recent evaluated nuclear data files, so uncertainty propagation calculations from nuclear data to reactivity are required for uncertainty quantification of reactivity.

When ones only consider static dependence of reactivity to nuclear data, uncertainty propagation calculations can be easily carried out with the adjoint-based procedure, in which sensitivity profiles of reactivity with respect to nuclear data are used. Static components of sensitivity profiles can be easily calculated by the classical perturbation theory. As described above, however, depletion dependence of reactivity on nuclear data should be also taken into account when systems consisting of spent nuclear fuels are considered. This depletion component of sensitivity profiles can be calculated by the depletion perturbation theory (DPT)[1,2], but it requires complicated numerical procedures and is computationally infeasible in calculations of large and complicated systems.

With this background and rapid advancement of computers, significant attentions have been paid to the stochastic-based procedure for uncertainty propagation calculations, and a lot of works with it have been carried out in recent years[3-5]. This procedure can treat large and complicated systems, and is free from the first-order approximation generally introduced to the adjoint-based procedure. There are, however, several drawbacks in the stochastic-based procedure, such as inherent statistical uncertainties and relatively long computation time.

In comparison with the stochastic-based procedure, the adjoint-based procedure does not include any statistical uncertainties. Through various kinds of analyses with sensitivity profiles and component decomposition of total uncertainties, physical investigation and consideration on numerical results become possible. Thus the adjoint-based procedure is 
still efficient and can be a complementary tool of the stochastic-based procedure.

Our research group has worked on development of numerical tools for uncertainty quantification of reactor physics parameters which are related to nuclear fuel depletion. On depletion-related reactor physics parameters of light water reactors, we have quantified those uncertainties induced by reaction cross sections for single cell problems[6]. We have used a DPT-based numerical module implemented to a reactor physics code system CBZ, which is under development at our research group. This DPT-based module has been extended to multi-cell problems[7], and DPT for fuel depletion calculations with the predictor-corrector method has been established[8]. With this new capability of CBZ, it is possible to perform uncertainty quantification calculations for multi-cell problems including burnable absorbers, which are much more realistic than single cell problems.

In the present paper, we quantify nuclear data-induced uncertainty of infinite neutron multiplication factors $\left(k_{\infty}\right)$ of a light water reactor (LWR) multi-cell including burnable absorbers during depletion with a new DPT-based module of CBZ and the recent version of JENDL nuclear data libraries, and compare the obtained results with those in a single cell. Physical interpretation of numerical results is also attempted by virtue of specific feature of the adjoint-based procedure. Our previous work has considered uncertainties only of reaction cross sections of actinoid nuclides[6], but the present work considers not only uncertainties of reaction cross sections but also those of fission yields and radioactive decay data. JENDL-4.0 does not contain any covariance information on FP nuclides[9], so assumption of $10 \%$ uncertainty with full energy correlation is introduced, and effect of FP nuclides on nuclear data-induced uncertainty is quantified. For criticality safety management of spent nuclear fuels, ones also should calculate $k_{\infty}$ uncertainties of systems after specific cooling period, so difference between results without any cooling and those with cooling is also discussed.

The present paper is organized as follows. Section 2 briefly describes theoretical back- 
grounds of uncertainty quantification with the adjoint-based procedure. In the present study, we employ not an original detail burnup chain, in which all the nuclides given in evaluated nuclear data files are taken into account, but a simplified chain consisting of a limited number of nuclides, so covariance matrices specific for a simplified chain are required. Section 3 describes a procedure to generate covariance matrices of a simplified chain. Numerical conditions and results are presented in Sec. 4, and all these are summarized in Sec. 5.

\section{Brief description of uncertainty quantification with the adjoint-based pro- cedure}

Here we consider $k_{\infty}$ at burnup period $i, k_{\infty, i}(i=1,2, \ldots, I)$, and nuclear data $\sigma_{j}(j=$ $1,2, \ldots, J)$. Sensitivity coefficient of $k_{\infty, i}$ with respect to $\sigma_{j}, S_{j}^{i}$, is defined as

$$
S_{j}^{i}=\frac{d k_{\infty, i}}{d \sigma_{j}} \cdot \frac{\sigma_{j}}{k_{\infty, i}}
$$

A derivative of $k_{\infty, i}$ to $\sigma_{j}$ can be decomposed into

$$
\frac{d k_{\infty, i}}{d \sigma_{j}}=\frac{\partial k_{\infty, i}}{\partial \sigma_{j}}+\sum_{l}\left(\frac{\partial k_{\infty, i}}{\partial N_{i, l}}\right)\left(\frac{d N_{i, l}}{d \sigma_{j}}\right),
$$

where $N_{i, l}$ is a number density of nuclide $l$ at burnup $i$. The first term of the right hand side of Equation (2) is referred to as the static term and the second term is as the depletion term in the present paper. Partial derivative of $k_{\infty, i}$ to $\sigma_{j}$ or $N_{i, l}$ can be easily calculated by the classical perturbation theory, and that of $N_{i, l}$ to $\sigma_{j}$ is calculated by DPT. Interested readers on DPT can refer papers cited in the reference list.

Sensitivity coefficients can be written in a matrix form as

$$
\mathbf{S}=\left(\begin{array}{cccc}
S_{1}^{1} & S_{1}^{2} & \cdots & S_{1}^{I} \\
S_{2}^{1} & S_{2}^{2} & \cdots & S_{2}^{I} \\
\vdots & \vdots & \ddots & \vdots \\
S_{J}^{1} & S_{J}^{2} & \cdots & S_{J}^{I}
\end{array}\right)
$$

If we denote to a covariance matrix of nuclear data as $\mathbf{V}_{\sigma}$, which is a $J \times J$ square matrix, 
an uncertainty propagation equation from $\sigma$ to $k_{\infty}$ can be written as

$$
\mathbf{V}_{k}=\mathbf{S}^{T} \mathbf{V}_{\sigma} \mathbf{S}
$$

where $\mathbf{V}_{k}$ is a covariance matrix of $k_{\infty, i}$, which is a $I \times I$ square matrix, and a superscript $T$ is used for a transpose of matrix. This is the well-known sandwich formula, and this equation is used in the following calculations.

\section{Covariance matrices generation for a simplified burnup chain}

In the present calculations, we take into account uncertainties of the following nuclear data: reaction cross sections, fission yields, decay half-lives and decay branching ratios. Those except reaction cross sections depend on a nuclide burnup chain used. When all the nuclides given in evaluated nuclear data files are explicitly considered in a burnup chain, covariance data originally provided in evaluated files can be directly used. Generally over 1,000 FP nuclides are provided in evaluated files, so burnup calculations with a burnup chain consisting of all these nuclides require significant computational burden, and simplified burnup chains consisting of several tens or around one hundred nuclides are generally preferred. When simplified burnup chains are used, covariance data of fission yields and decay data should be recalculated from original covariance data provided in evaluated nuclear data files.

Attentions should be paid on covariance data of fission yields and decay branching ratios when a simplified burnup chain is used. On fission yields, covariance data of independent fission yields can be applied when an original detail burnup chain is used. On the other hand, when a simplified burnup chain is used, covariance data of partiallycumulative fission yields, which are sum of independent fission yields of nuclides, which are positioned at a upstream chain and neglected in a simplified chain, should be prepared. Figure 1 shows a portion of an original burnup chain related to xenon-131 generation. If tellurium-131 is neglected in a simplified chain, fission yield of iodine- 131 in this chain 
should be sum of independent yields of iodine-131 and tellurium-131. This is an example of partially-cumulative fission yields. On decay branching ratios, it should be emphasized that those uncertainties in original data can become those of decay branching ratios, fission yields and reaction branching ratios in a simplified chain. As shown in Figure 1, antimony-131 has two decay channels and each of branching ratios has its uncertainty. If antimony-131 is neglected in a simplified chain, uncertainties of decay branching ratios should be considered as uncertainties of partially-cumulative fission yields of ground and meta-stable states of tellurium-131. These uncertainties of partially-cumulative fission yields induced by antimony-131 decay branching ratios should be correlated among different fissile nuclides, so a unique fully-correlated covariance matrix of partially-cumulative fission yields induced by decay branching ratios should be prepared in uncertainty quantification calculations with a simplified chain.

[Figure 1 about here.]

Uncertainties of reaction branching ratios in a simplified chain induced by uncertainties of decay branching ratios in original data are also noted. Figure 2 shows a portion of original and simplified chains related to gadolinium-152 generation. Meta-stable state of europium152 has two decay channels and each of branching ratios has its uncertainty. When this nuclide is neglected in a simplified chain, these uncertainties should be considered as uncertainties of $(n, \gamma)$ reaction branching ratios of europium-151.

[Figure 2 about here.]

It is possible to analytically obtain covariance matrices of fission yields, decay branching ratios and reaction branching ratios in a simplified chain, but its derivation and implementation to a computer code might be cumbersome. In the present study, the stochastic-based procedure is adopted to these covariance data generation; sets of samples about burnup-related nuclear data are generated stochastically in accordance with their covariance data, simplified chains based on these nuclear data sets are prepared, and 
then covariance matrices of the above nuclear data in a simplified chain are calculated.

\section{Numerical results}

\subsection{Numerical procedure}

All the calculations are carried out by a reactor physics code system CBZ. Numerical procedure of nuclear fuel depletion calculations with CBZ is briefly described below.

Resonance self-shielding calculations, i.e., effective cross sections calculations, are carried out in a single cell with reflective boundary conditions with the advanced Bondarenko method[10]. A 107-group library based on JENDL-4.0 is used. The resonance self-shielding calculations yield 107-group cross sections which are used in subsequent neutron flux and fuel depletion calculations. Neutron transport equation is solved by a MEC module of CBZ, which is based on the method of characteristics for spatial and angle discretization. Nuclides depletion equation is solved by the matrix exponential method, and the minimax polynomial approximation method is used to calculate the matrix exponential[11]. A simplified burnup chain consisting of 138 FP nuclides, which are chosen by specific algorithm to reproduce number densities of some important nuclides after depletion[12], is used. Fission yields data and radioactive decay data of FP nuclides are taken from an updated version of JENDL FP yields data file-2011(JENDL/FPY-2011) and FP decay data file-2011(JENDL/FPD-2011)[13], in which nuclear data of over 1,300 FP nuclides are evaluated. In calculations of a multi-cell including burnable absorbers, an optimallyweighted predictor-corrector method is adopted to reduce time discretization error[14].

It has been widely recognized that there are strong negative correlations in independent fission yields among different nuclides belonging to a single mass chain, and these correlations have significant influence on uncertainty quantification calculations for nuclide number densities after depletion even though such correlation data are not provided in current evaluated nuclear data files[15]. We apply the following method of estimating 
covariance among different nuclides in a single mass chain, which has been proposed by Devillers[16]:

$$
\begin{aligned}
& \bar{V}_{i, i}=V_{i}\left(1-\frac{V_{i}}{V_{M Y}+\sum_{j=1}^{J} V_{j}}\right), \\
& \bar{V}_{i, j}=-\frac{V_{i} V_{j}}{V_{M Y}+\sum_{j=1}^{J} V_{j}},
\end{aligned}
$$

where $\bar{V}_{i, j}$ are covariance between fission yields of nuclides $i$ and $j, V_{i}$ is variance of fission yield of nuclide $i$ originally given in JENDL/FPY-2011, $V_{M Y}$ is variance of mass yield, and $J$ denotes the number of nuclides in the same mass chain.

Since covariance data are not given for some nuclear data in JENDL/FPD-2011, we assume that these nuclear data have $100 \%$ relative uncertainty as a previous work about decay heat uncertainty calculations done by Katakura[13]. Branching ratio uncertainties should have strong negative correlations among different decay paths of a single nuclide because of the normalization condition. This is also taken into consideration.

\subsection{Problem specifications}

A PWR-simulated $\mathrm{UO}_{2}$ fuel single cell and a $3 \times 3$ multi-cell consisting of this single cell with reflective boundary conditions are considered. Geometric configuration of the multi-cell is shown in Figure 3 .

[Figure 3 about here.]

Uranium-235 enrichment of the $\mathrm{UO}_{2}$ fuel pins is $3.4 \mathrm{wt} \%$. Geometric specification and initial nuclide number densities are taken from the reference[17]. At the center position of the multi-cell, a gadolinium-bearing $\mathrm{UO}_{2}$ fuel pin, whose gadolinium concentration is $10 \mathrm{wt} \%$ and uranium-235 enrichment is $2.63 \mathrm{wt} \%$, is located. This gadolinium-bearing pin is divided into eight ring-type regions, and nuclides depletion calculations are carried out 
in each region. On the other normal fuel pins (pin 2 and pin 3), a whole fuel pellet region is treated as one depletion region. The same condition is adopted to a single cell calculation. Neutron flux is normalized by linear power density per one fuel pin of $179 \mathrm{~W} / \mathrm{cm}$. Infinite neutron multiplication factors during fuel depletion of a single cell and a $3 \times 3$ multi-cell are shown in Figure 4.

[Figure 4 about here.]

\subsection{Verification of covariance matrices for a simplified burnup chain}

In order to verify covariance matrices for a simplified chain generated by the abovedescribed procedure, variance of nuclides number densities after fuel depletion is calculated by a simplified chain and its covariance matrices. Reference values of variance are obtained from sensitivity profiles calculated with an original detail chain and covariance data given in the evaluated nuclear data. A simplified chain consisting of $138 \mathrm{FP}$ nuclides has been proposed so as to reproduce number densities of $33 \mathrm{FP}$ nuclides, which have relatively large impact on reactivity[12], so number densities of these $33 \mathrm{FP}$ nuclides are concerned in this verification calculation. Three sets of covariance matrices of a simplified chain are generated from a hundred, a thousand, and ten thousands samples. Table 1 shows root-mean-square and maximum relative errors in variance of number densities after $40 \mathrm{GWD} / \mathrm{t}$ of burnup in the single cell. Number densities of nuclide in the parenthesis gives the maximum error in variance. Variances are separately evaluated as fission yield(FY)-induced one and decay branching ratio(DBR)-induced one. On fission yieldinduced variance, RMS and maximum relative errors of simplified chain results become small as the number of samples increases. On the other hand, maximum relative error of decay branching ratio-induced variance is still large (7.6\%) even if ten thousands samples is used. Through further investigation on this large error observed in xenon-131 number density, it has been found that uncertainty of decay branching ratio of antimony-131 is not 
reproduced well with a simplified chain. In our procedure of simplified burnup chain construction, unimportant nuclides, which are neglected in a simplified chain, are identified by observing adjoint number densities (or important functions) of these nuclides to target quantity. However, antimony-131 is neglected in the present simplified chain because of similarity in adjoint number density between antimony-131 and tellurium-131, which is different criteria in our important nuclides identification process[12]. We do not yet sufficiently understand the reason why antimony-131 branching ratio-induced variance is not well reproduced. Note that relative standard deviation in xenon-131 number density induced by decay branching ratio is about $0.003 \%$, so impact of this relatively large error of $7.6 \%$ is insignificant. The second largest maximum error induced by decay branching ratio is 0.028 in variance of Mo-95 number densities. In our calculations, covariance matrices generated from 10,000 samples are used.

In order to further verify our procedure of covariance data generation, antimony-131 is added to the FP-138 chain and the same uncertainty propagation calculations are carried out. Results of this FP-139 chain are also shown in Table 1. Maximum errors in variance induced by decay branching ratios become small as the number of samples increases. This result also verifies our procedure to generate covariance matrices of a simplified burnup chain.

[Table 1 about here.]

\subsection{Uncertainty quantification of neutron multiplication factors during nuclear fuel depletion}

\subsubsection{Comparison between a single cell and a multi-cell including burnable absorber}

Nuclear data-induced uncertainties of $k_{\infty}$ during fuel depletion of a single cell and a $3 \times 3$ multi-cell are shown in Figure 5. Although slight difference is observed between these two uncertainties around $20 \mathrm{GWD} / \mathrm{t}$ of burnup, uncertainties and their dependence on 
burnup are similar to each other. Figure 6 shows correlation matrix of these uncertainties; this matrix has a block structure and the left-bottom and right-top blocks are sub-matrices about that of a single cell and that of a multi-cell, respectively, and non-diagonal submatrices are correlation between these two components.

Strong positive correlation between uncertainties of the single cell and the multi-cell at same burnup is observed. Strong correlations among uncertainties at different burnup periods are also observed.

[Figure 5 about here.]

[Figure 6 about here.]

Nuclear data-wise uncertainties are also calculated, and it is found that reaction cross sections of actinoids are dominant contributors to total uncertainties, and that other components such as fission yields and decay data are negligible. Uncertainties of $k_{\infty}$ induced by fission yield, half-life and decay branching ratio uncertainties are shown in Figure 7. Among them fission yield-induced uncertainties are the largest, but those are less than $0.03 \%$ and much less than reaction cross section-induced uncertainties. These small fission yield-induced uncertainties are consistent with the previous works based on the stochastic-based procedure[15, 18].

[Figure 7 about here.]

Figure 8 shows nuclide-wise reaction cross section-induced uncertainties of $k_{\infty}$ during fuel depletion. In this figure, contributions of five dominant nuclides are presented. With burnup, uranium-235-induced uncertainties decrease and plutonium isotopes-induced uncertainties increase because of changes in their inventories. Decreases of uranium-238induced uncertainties with burnup can be easily understood by observing sensitivity profiles during fuel depletion. Figure 9 shows energy-integrated sensitivities of $k_{\infty}$ to uranium-238 capture cross section during depletion. Static terms of sensitivity are constantly negative, but positive depletion terms increase with burnup. This is due to conver- 
sion effect from uranium-238 to plutonium-239 via neutron capture reaction of uranium238; increase of uranium-238 capture cross section promotes nuclear fuel conversion and makes $k_{\infty}$ large. This is positive contribution to sensitivity, and cancels static contribution out. In $k_{\infty}$ uncertainties in the multi-cell, slight fluctuation is observed around $15 \mathrm{GWD} / \mathrm{t}$, and this can be interpreted as follows; if uranium-238 capture cross section is increased, this makes plutonium-239 inventories large because of promotion of conversion. Plutonium-239 absorbs thermal neutrons strongly, so neutron flux energy spectrum shifts to high energy and thermal neutron captures by gadolinium-155 and -157 are reduced. Thus these gadolinium isotopes still remain in a fuel, so $k_{\infty}$ becomes small. This is negative contribution to sensitivity and this makes dips in the depletion term around $15 \mathrm{GWD} / \mathrm{t}$ in a $3 \times 3$ multi-cell as shown in Figure 9 .

[Figure 8 about here.]

[Figure 9 about here.]

\subsubsection{Effect of uncertainty of neutron capture cross sections of fission product nuclides}

In the preceding calculations, uncertainties of reaction cross sections of FP nuclides have not been considered since no evaluations have been made on covariance data of these nuclides in JENDL-4.0. However, reaction cross sections of FP nuclides should be uncertain and those must be taken into account to obtain more reliable uncertainty of $k_{\infty}$. Here we assume fully-energy-correlated $10 \%$ uncertainty to neutron capture cross sections of all the FP nuclides considered in our simplified chain. It is reported in Ref.[19] that calculation-to-experimental values about material sample worth of several fission products such as Mo-95 and Ru-101 range from 0.87 to 1.17, so the present assumption of $10 \%$ uncertainty is realistic. In order to distinguish contributions of burnable absorbers from FP nuclides, another case in which $10 \%$ uncertainties are assumed to all the FPs except gadolinium-155 and -157 is also prepared. Uncertainties of $k_{\infty}$ induced by capture cross 
sections of FP nuclides are shown in Figure 10. Uncertainties of $k_{\infty}$ reach about $0.2 \%$ at $40 \mathrm{GWD} / \mathrm{t}$ in both cases. Those uncertainties are much smaller than those induced by reaction cross sections of actinoids. In the multi-cell, fluctuations in $k_{\infty}$ uncertainties are observed around $15 \mathrm{GWD} / \mathrm{t}$. Figure 11 shows energy-integrated sensitivities of $k_{\infty}$ to gadolinium-155 and -157 capture cross sections. As $k_{\infty}$ sensitivity to uranium-238 capture cross sections, cancellation between static and depletion terms can be observed. Depletion term is positive because increase in capture cross sections of these gadolinium isotopes enhances depletion of these isotopes. Observed burnup dependence of $k_{\infty}$ uncertainty in the multi-cell can be understood from these sensitivity profiles with burnup.

[Figure 10 about here.]

[Figure 11 about here.]

In order to specify FP nuclides that are important in uncertainty quantification of $k_{\infty}$ during fuel depletion, energy-integrated sensitivities of $k_{\infty}$ to capture cross sections during depletion are shown in Figure 12. Ten FP nuclides, whose sensitivities are large at $40 \mathrm{GWD} / \mathrm{t}$, are chosen here. In addition to energy-integrated sensitivities, energy spectra of sensitivities at $40 \mathrm{GWD} / \mathrm{t}$ are also shown in Figure 13. These information might be important for future evaluation of covariance data of FP nuclides nuclear data.

[Figure 12 about here.]

[Figure 13 about here.]

\subsubsection{Effect of cooling time}

Reactivity of spent nuclear fuels change with cooling period because of radioactive decay of unstable nuclides. Here $k_{\infty}$ uncertainties after several different burnup and cooling periods of 5 and 10 years are calculated. Figure 14 shows nuclear data-induced uncertainties of $k_{\infty}$. Note that uncertainties of capture cross sections of FP nuclides are not considered. Changes in $k_{\infty}$ uncertainties due to different cooling period are insignifi- 
cant when JENDL-4.0 is used. These changes are due to radioactive decay of plutonium241. Figure 15 shows $k_{\infty}$ uncertainties induced by plutonium-241 and americium-241 nuclear data. Because of decay of plutonium-241 with half-life of 14.29 years, contribution of plutonium-241 decreases and that of americium-241, which is daughter nuclide of plutonium-241, increases as cooling period increases. Gradients of $k_{\infty}$ uncertainties to burnup are steeper in plutonium-241 than in americium-241. This difference can be understood by observing energy-integrated sensitivities of $k_{\infty}$ to capture cross sections of these nuclides, which are shown in Figure 16. Because most of americium-241 is generated during cooling period, depletion term is small in sensitivity to americium-241 capture cross section.

[Figure 14 about here.]

[Figure 15 about here.]

[Figure 16 about here.]

Contributions of capture cross sections of FP nuclides are also considered. Figure 17 shows $k_{\infty}$ uncertainties after specific cooling period induced by FP nuclides nuclear data. Fully-energy-correlated $10 \%$ uncertainty is assumed as the preceding section. Figure 18 shows energy-integrated sensitivity of $k_{\infty}$ to capture cross sections of xenon-135, gadolinium-155 and europium-155. When cooling period is considered, xenon-155 contribution becomes small because of its short half-life of 9.14 hours. Also due to radioactive decay of europium-155 with half-life of 4.753 years, gadolinium-155, which is strong neutron absorber, is produced and this contributes to total uncertainties.

[Figure 17 about here.]

[Figure 18 about here.] 


\section{Conclusion}

We have quantified nuclear data-induced uncertainties of infinite neutron multiplication factors during fuel depletion in a single cell and a $3 \times 3$ multi-cell including burnable absorbers. Uncertainties of reaction cross sections of actinoids and fission product nuclides, fission yields, decay half-lives and decay branching ratios have been taken into account. Uncertainties propagation calculations have been carried out with the adjoint-based procedure, and required sensitivity profiles of infinite multiplication factors with respect to these nuclear data have been efficiently calculated by the depletion perturbation theory. Covariance matrices for fission yields and decay data in a simplified chain have been successfully generated by the stochastic-based procedure. Infinite neutron multiplication factors uncertainties of about $0.6 \%$ have been obtained during fuel depletion, and it has been shown that actinoids reaction cross sections are dominant contributors. Nuclide-wise decomposition of the uncertainties and observation of component-wise sensitivity profiles have provided physical interpretations.

By virtue of the adjoint-based procedure, several parametric surveys have also been conducted. Contributions of uncertainties in fission products nuclides have been quantified, and important nuclides and energy ranges have been identified for further evaluation of nuclear data of FP nuclides. Effect of cooling period on infinite multiplication factor uncertainties has been also discussed.

\section{Acknowledgement}

This work is supported by the secretariat of the nuclear regulation authority of Japan.

\section{References}

[1] Gandini A. A method of correlation of burnup measurements for physics prediction of fast powerreactor life. Nucl Sci Eng. 1969; 38: 1-7. 
[2] Williams ML. Development of depletion perturbation theory for coupled neutron/nuclide fields. Nucl Sci Eng. 1979; 70: 20-36.

[3] Kawano T, Hanson KM, Frankle S, Talou P, Chadwick MB, Little RC. Evaluation and propagation of the ${ }^{239} \mathrm{Pu}$ fission cross-section uncertainties using a Monte Carlo technique. Nucl Sci Eng. 2006; $153: 1-7$

[4] Rochman D, Koning AJ, van der Marck SC. Uncertainties for criticality-safety benchmarks and $k_{\text {eff }}$ distributions. Ann Nucl Energy 2009; 36:810-831.

[5] Yamamoto A, Kinoshita K, Watanabe T, Endo T, Kodama Y, Ohoka Y, Ushio T, Nagano H. Uncertainty quantification of LWR core characteristics using random sampling method. Nucl Sci Eng. 2015; 181:160-174.

[6] Chiba G, Tsuji M, Narabayashi T. Uncertainty quantification of neutronic parameters of light water reactor fuel cells with JENDL-4.0 covariance data. J Nucl Sci Technol. 2013; 50: 751-760.

[7] Chiba G, Kawamoto Y, Narabayashi T. Development of a fuel depletion sensitivity calculation module for multi-cell problem in a deterministic reactor physics code system CBZ. Ann Nucl Energy. 2016; 96: 277-286.

[8] Chiba G. Perturbation theory for nuclear fuel depletion calculations with predictor-corrector method. J Nucl Sci Technol. 2018; 55: 290-300.

[9] Shibata K, Iwamoto O, Nakagawa T, Iwamoto N, Ichihara A, Kunieda S, Chiba S, Furukawa K, Otuka N, Ohsawa T, Murata T, Matsunobu H, Zukaran A, Kameda S, Katakura J. JENDL-4.0: A new library for nuclear science and technology. J Nucl Sci Technol. 2011; 48:1-30.

[10] Chiba G, Narabayashi T. Advanced Bondarenko method for resonance self-shielding calculations in deterministic reactor physics code system CBZ. Ann Nucl Energy. 2016; 96: 277-286.

[11] Kawamoto Y, Chiba G, Tsuji M, Narabayashi T. Numerical solution of matrix exponential in burn-up equation using mini-max polynomial approximation. Ann Nucl Energy. 2015; 80: 219-224.

[12] Chiba G, Tsuji M, Narabayashi T, Ohoka Y, Ushio T. Important fission product nuclides identification method for simplified burnup chain construction, J Nucl Sci Technol. 2015; 52: 953-960. 
[13] Katakura J. JENDL FP decay data file 2011 and fission yields data file 2011. Japan: Japan Atomic Energy Agency; 2011, JAEA-Data/Code 2011-025.

[14] Okumura S, Chiba G. Development of nuclear fuel depletion calculation capability for LWR fuel assembly in reactor physics code system CBZ. Proc. Reactor Physics Asia 2017; 2017 Aug 24-25; Chengdu (China).

[15] Cabellos O, Piedra D, Diez CJ. Impact of the fission yield nuclear data uncertainties in the pin-cell burn-up OECD/NEA UAM benchmark. Proc. Int. Conf. on Physics of Reactors; 2014 Sep 28- Oct 3 ; Kyoto (Japan).

[16] Devillers, C., 1977. The importance of fission product nuclear data in reactor design and operation, IAEA Panel, Petten.

[17] Katakura J, Kataoka M, Suyama K, Jin T, Ohki S. A set of ORIGEN2 cross section libraries based on JENDL-3.3 library: ORLIBJ33. Japan: Japan Atomic Energy Research Institute; 2004, JAERIData/Code 2004-015.[in Japanese]

[18] Fiorito L, Stankovskiy A, Van den Eynde G, Diez C.J, Cabellos O, Labeau P.E. Generation of fission yield covariances to correct discrepancies in the nuclear data libraries. Ann Nucl Energy. 2016; 88: $12-23$.

[19] Aliberti G, Palmiotti G, Salvatores M, Kim T.K, Taiwo T.A, Anitescu M, Kodeli I, Sartori E, Bosq J.C, Tommasi J. Nuclear data sensitivity, uncertainty and target accuracy assessment for future nuclear systems. Ann Nucl Energy. 2006; 33: 700-733. 
Table 1 Root-mean-square (RMS) and maximum relative errors in variance of number densities after fuel depletion

\begin{tabular}{cccccc}
\hline $\begin{array}{c}\text { FP } \\
\text { chain }\end{array}$ & $\begin{array}{c}\text { Number of } \\
\text { samples }\end{array}$ & \multicolumn{2}{c}{ FY-induced } & \multicolumn{2}{c}{ DBR-induced } \\
FP-138 & 100 & 0.102 & $0.316\left(\mathrm{Pm}-149^{*}\right)$ & 0.493 & $2.731(\mathrm{Xe}-131)$ \\
& 1,000 & 0.035 & $0.112(\mathrm{Sm}-150)$ & 0.034 & $0.088(\mathrm{Pd}-107)$ \\
& 10,000 & 0.013 & $0.025(\mathrm{Eu}-155)$ & 0.017 & $0.076(\mathrm{Xe}-131)$ \\
\hline FP-139 & 100 & 0.102 & $0.315(\mathrm{Pm}-149)$ & 0.132 & $0.482(\mathrm{Pd}-107)$ \\
& 1,000 & 0.035 & $0.113(\mathrm{Sm}-150)$ & 0.032 & $0.088(\mathrm{Pd}-107)$ \\
& 10,000 & 0.013 & $0.026(\mathrm{Eu}-155)$ & 0.011 & $0.028(\mathrm{Mo}-95)$ \\
\hline
\end{tabular}

* Number densities of this nuclide gives the maximum error in variance. 


\section{Figure Captions}

Figure 1 Burnup chain related to xenon-131 generation

Figure 2 Example of reaction branching ratio uncertainty in a simplified chain induced by decay branching ratio uncertainty in an original chain

Figure 3 Geometrical specification of 3x3 multi-cell including burnable neutron absorber

Figure 4 Infinite neutron multiplication factors during fuel depletion

Figure 5 Nuclear data-induced uncertainty of infinite neutron multiplication factors during fuel depletion

Figure 6 Correlation matrices of nuclear data-induced uncertainty of infinite neutron multiplication factors during fuel depletion

Figure 7 Component-wise nuclear data-induced uncertainty of $k_{\infty}$ during fuel depletion

Figure 8 Nuclide-wise reaction cross section-induced uncertainty of $k_{\infty}$ during fuel depletion

Figure 9 Energy-integrated sensitivity of $k_{\infty}$ with respect to uranium-238 capture cross sections

Figure 10 FP nuclides capture cross section-induced uncertainty of $k_{\infty}$ during fuel depletion 
Figure 11 Energy-integrated sensitivity of $k_{\infty}$ in $3 \times 3$ multi-cell with respect to gadolinium-155 and -157 capture cross sections

Figure 12 Energy-integrated sensitivity of $k_{\infty}$ in single cell with respect to capture cross sections of FP nuclides

Figure 13 Energy spectra of sensitivities of $k_{\infty}$ in a single cell with respect to capture cross sections of FP nuclides

Figure 14 Nuclear data-induced uncertainty of $k_{\infty}$ after specific cooling period

Figure 15 Nuclide-wise nuclear data-induced uncertainty of $k_{\infty}$ after specific cooling period

Figure 16 Energy-integrated sensitivity of $k_{\infty}$ after 5-year cooling with respect to capture cross sections of plutonium-241 and americium-241

Figure 17 FP nuclides nuclear data-induced uncertainty of $k_{\infty}$ after specific cooling period

Figure 18 Energy-integrated sensitivity of $k_{\infty}$ in single cell with respect to capture cross sections of FP nuclides 
J. Nucl. Sci. \& Technol.

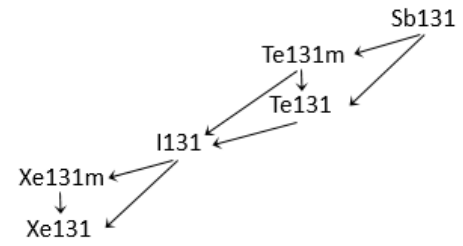

Figure 1 Burnup chain related to xenon-131 generation

\section{G.Chiba}

Uncertainty quantification of neutron multiplication factors of light water reactor fuels during depletion 
J. Nucl. Sci. \& Technol.

Article

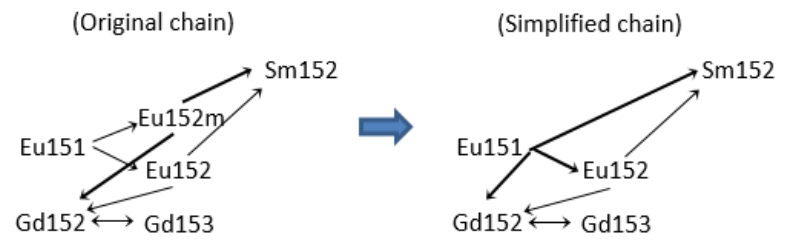

Figure 2 Example of reaction branching ratio uncertainty in a simplified chain induced by decay branching ratio uncertainty in an original chain

G.Chiba

Uncertainty quantification of neutron multiplication factors of light water reactor fuels during depletion 


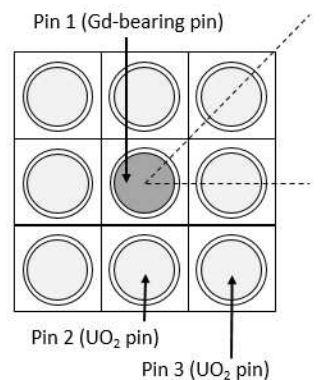

Figure 3 Geometrical specification of 3x3 multi-cell including burnable neutron absorber

\section{G.Chiba}

Uncertainty quantification of neutron multiplication factors of light water reactor fuels during depletion 


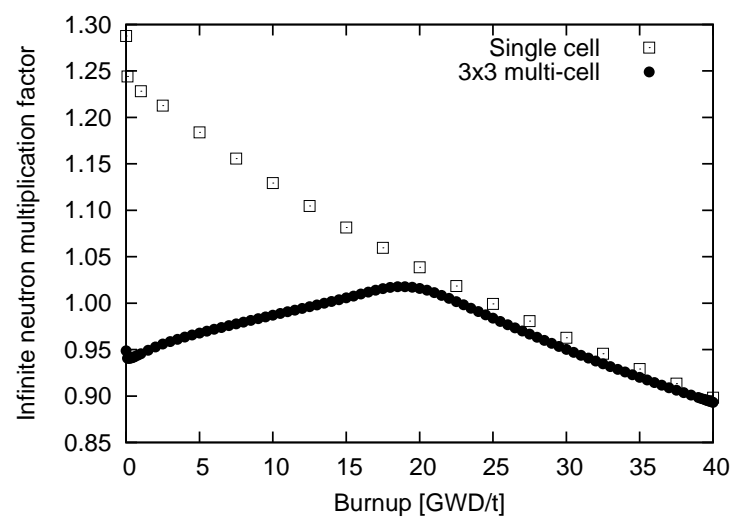

Figure 4 Infinite neutron multiplication factors during fuel depletion

\section{G.Chiba}

Uncertainty quantification of neutron multiplication factors of light water reactor fuels during depletion 


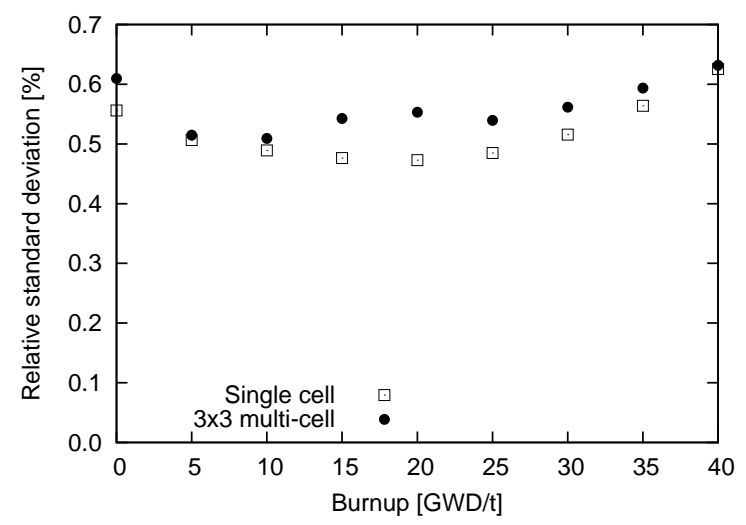

Figure 5 Nuclear data-induced uncertainty of infinite neutron multiplication factors during fuel depletion

G.Chiba

Uncertainty quantification of neutron multiplication factors of light water reactor fuels during depletion 


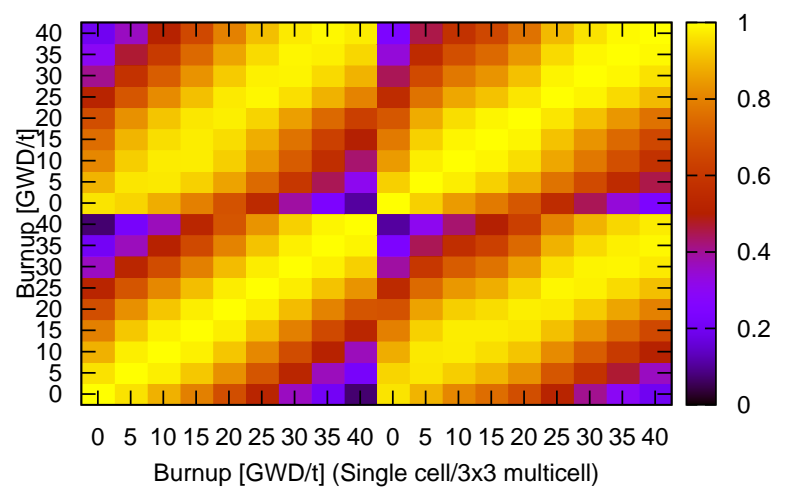

Figure 6 Correlation matrices of nuclear data-induced uncertainty of infinite neutron multiplication factors during fuel depletion

\section{G.Chiba}

Uncertainty quantification of neutron multiplication factors of light water reactor fuels during depletion 

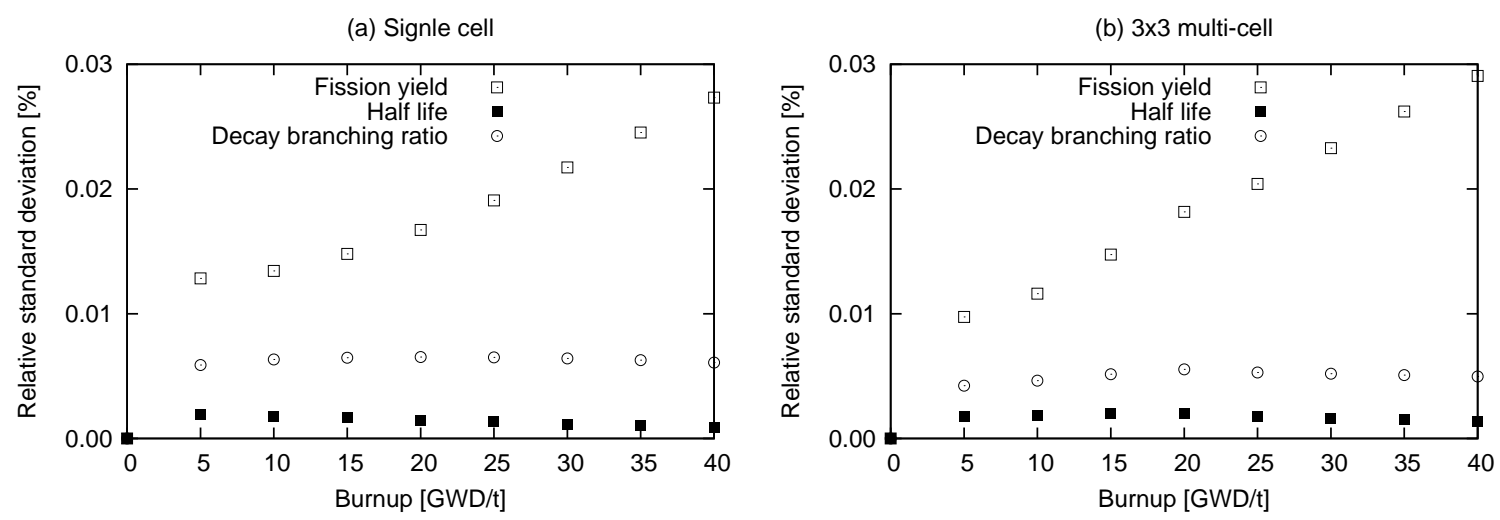

Figure 7 Component-wise nuclear data-induced uncertainty of $k_{\infty}$ during fuel depletion

\section{G.Chiba}

Uncertainty quantification of neutron multiplication factors of light water reactor fuels during depletion 
(a) Single cell

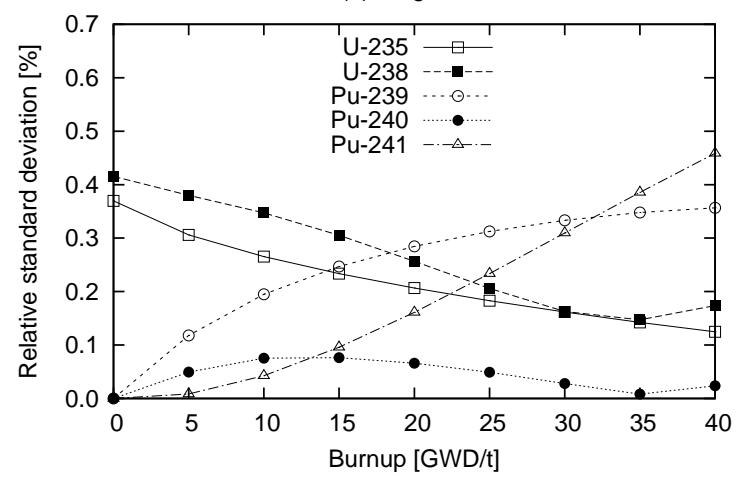

(b) $3 \times 3$ multi-cell

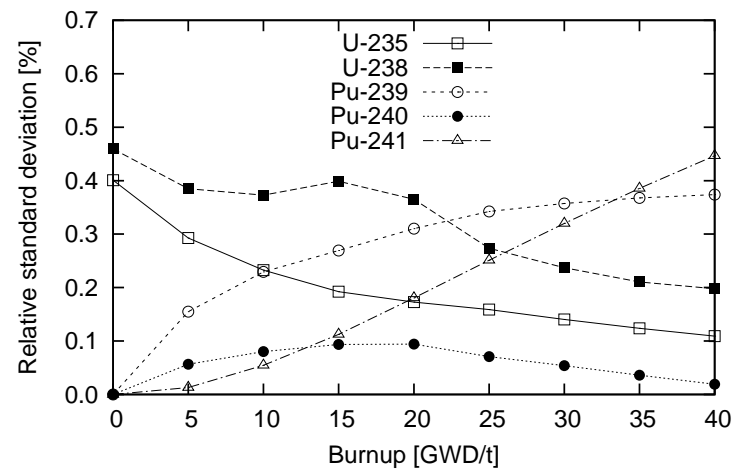

Figure 8 Nuclide-wise reaction cross section-induced uncertainty of $k_{\infty}$ during fuel depletion

\section{G.Chiba}

Uncertainty quantification of neutron multiplication factors of light water reactor fuels during depletion 
(a) Single cell

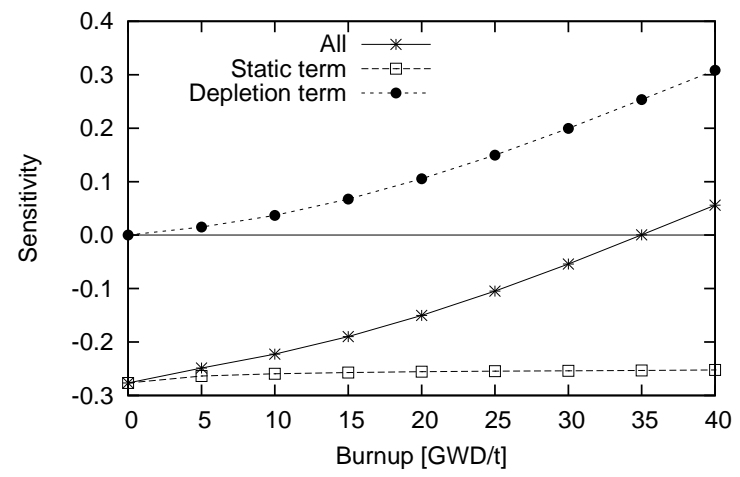

(a) $3 \times 3$ multi-cell

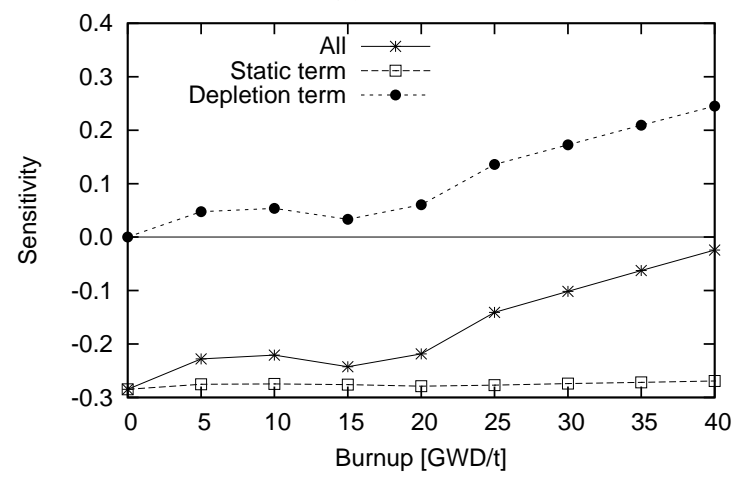

Figure 9 Energy-integrated sensitivity of $k_{\infty}$ with respect to uranium-238 capture cross sections

\section{G.Chiba}

Uncertainty quantification of neutron multiplication factors of light water reactor fuels during depletion 
(a) Single pincell

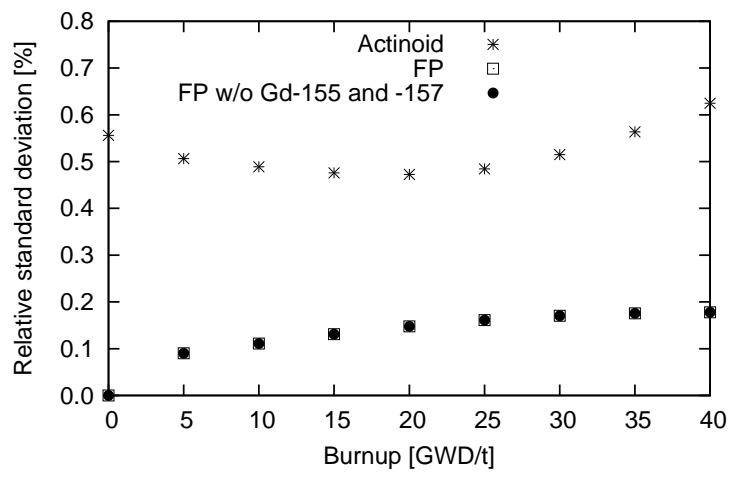

(b) $3 \times 3$ multi-cell

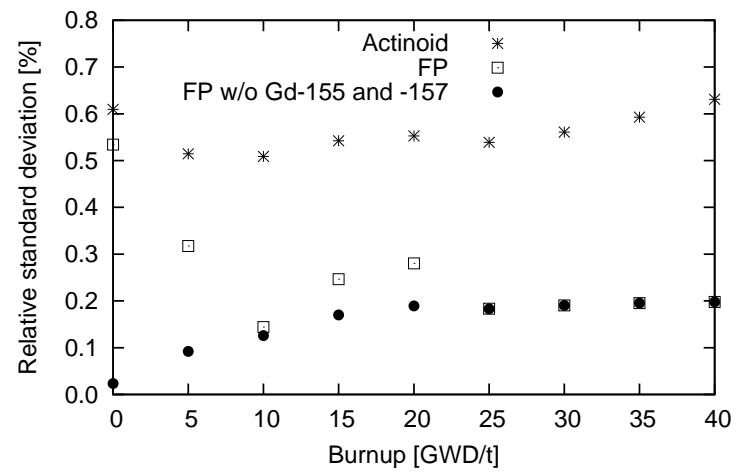

Figure $10 \mathrm{FP}$ nuclides capture cross section-induced uncertainty of $k_{\infty}$ during fuel depletion

\section{G.Chiba}

Uncertainty quantification of neutron multiplication factors of light water reactor fuels during depletion 

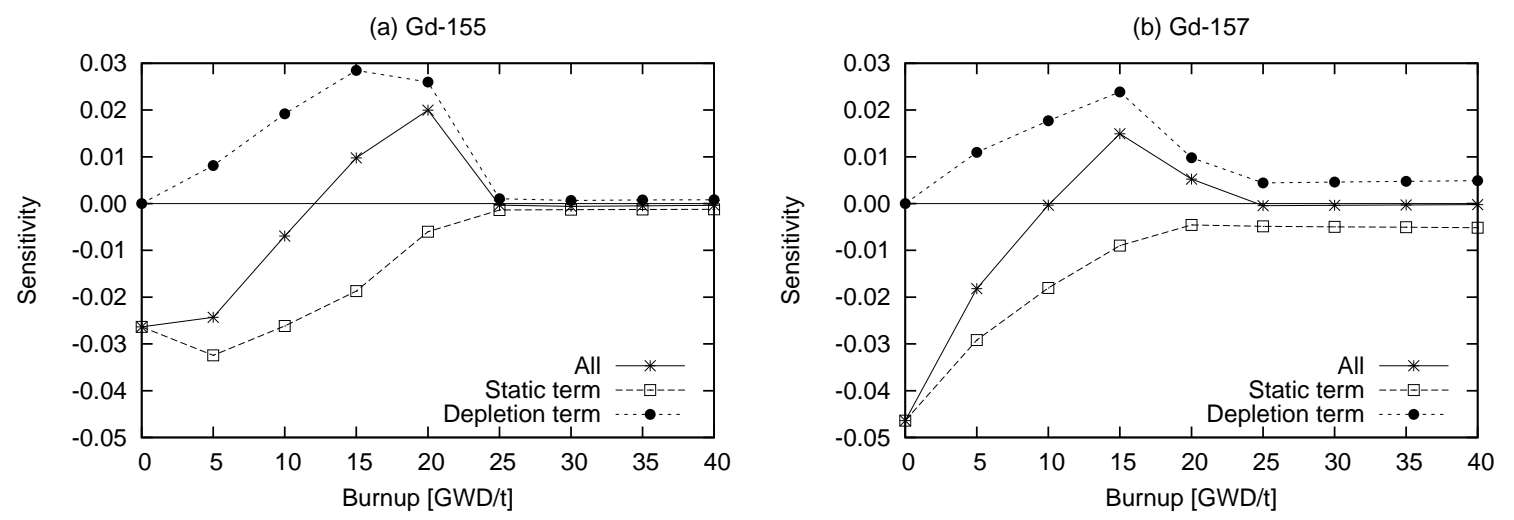

Figure 11 Energy-integrated sensitivity of $k_{\infty}$ in 3x3 multi-cell with respect to gadolinium-155 and -157 capture cross sections

G.Chiba

Uncertainty quantification of neutron multiplication factors of light water reactor fuels during depletion 

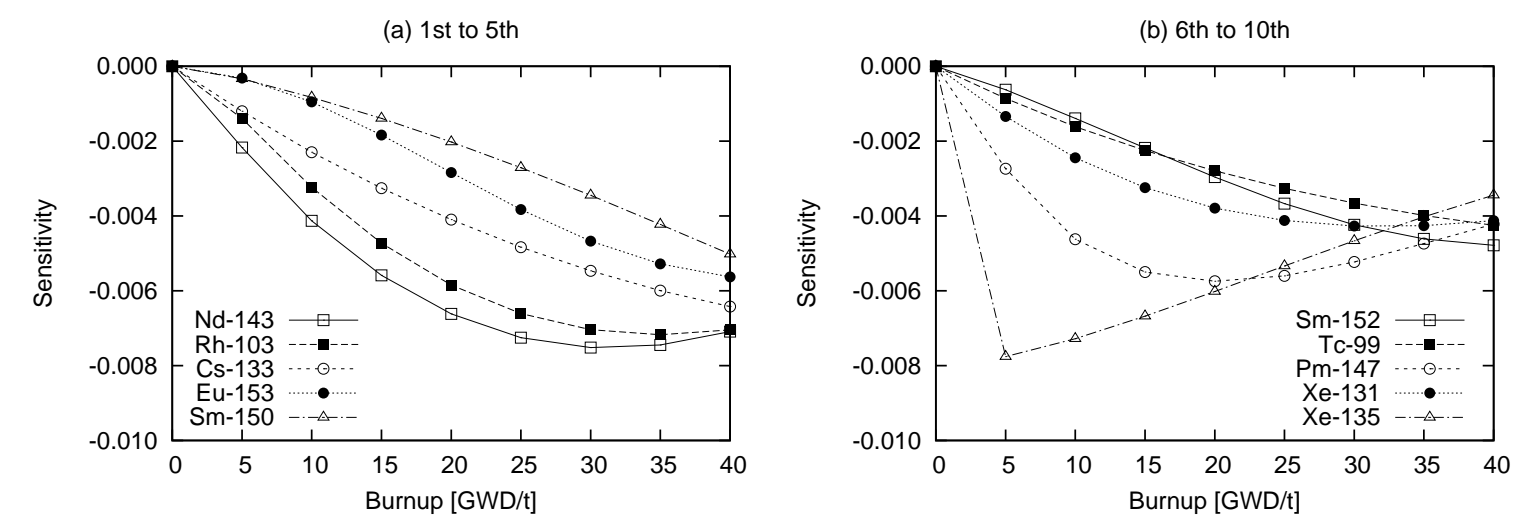

Figure 12 Energy-integrated sensitivity of $k_{\infty}$ in single cell with respect to capture cross sections of FP nuclides

G.Chiba

Uncertainty quantification of neutron multiplication factors of light water reactor fuels during depletion 
(a) 1 st to 5 th

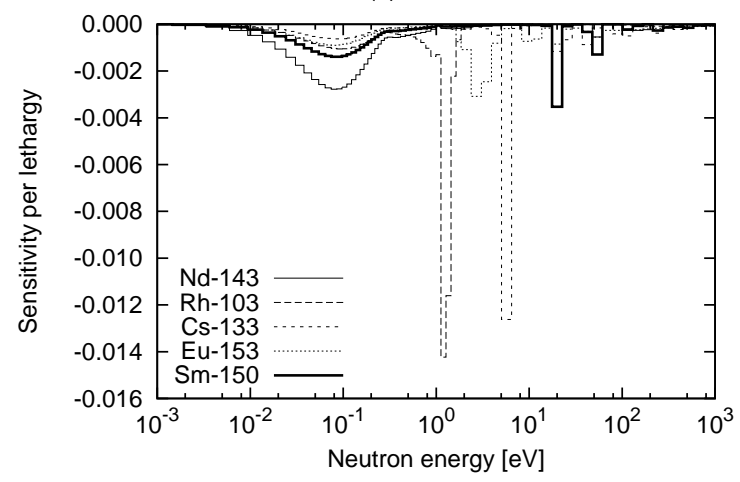

(b) 6th to 10 th

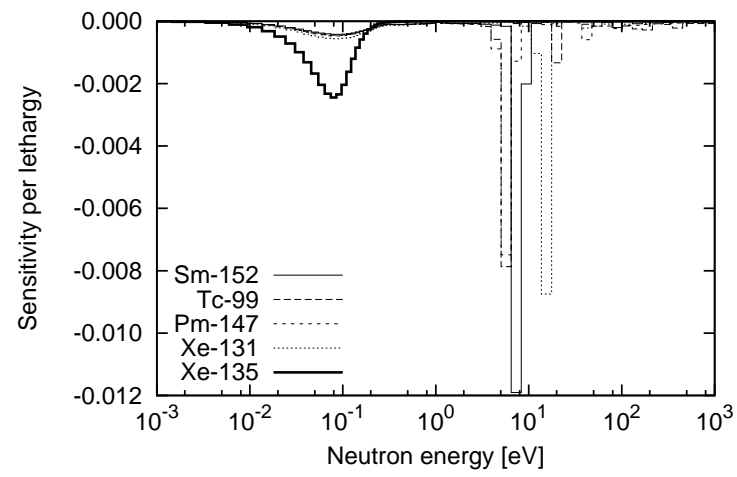

Figure 13 Energy spectra of sensitivities of $k_{\infty}$ in a single cell with respect to capture cross sections of FP nuclides

\section{G.Chiba}

Uncertainty quantification of neutron multiplication factors of light water reactor fuels during depletion 


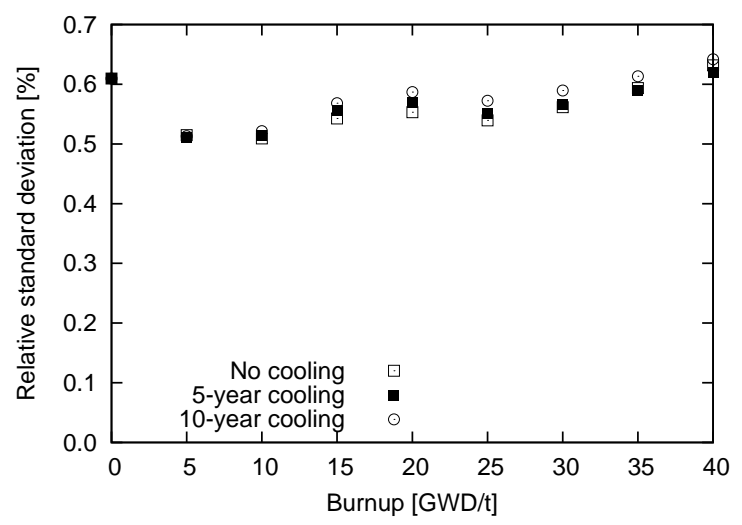

Figure 14 Nuclear data-induced uncertainty of $k_{\infty}$ after specific cooling period

\section{G.Chiba}

Uncertainty quantification of neutron multiplication factors of light water reactor fuels during depletion 
(a) Pu-241

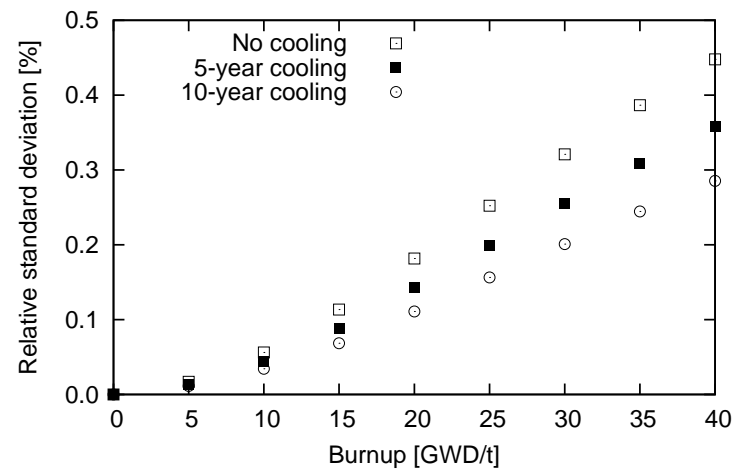

(b) Am-241

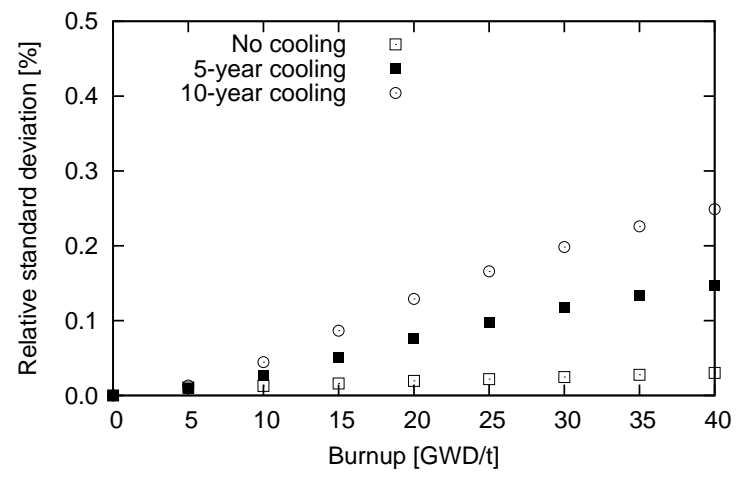

Figure 15 Nuclide-wise nuclear data-induced uncertainty of $k_{\infty}$ after specific cooling period

\section{G.Chiba}

Uncertainty quantification of neutron multiplication factors of light water reactor fuels during depletion 
(a) Pu-241

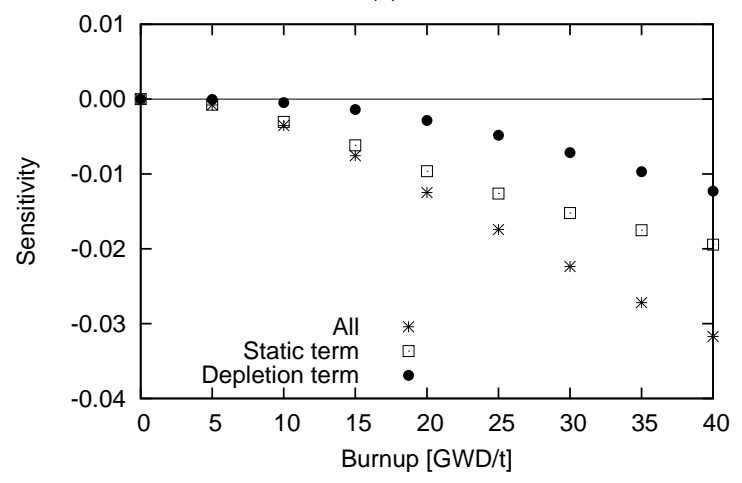

(b) Am-241

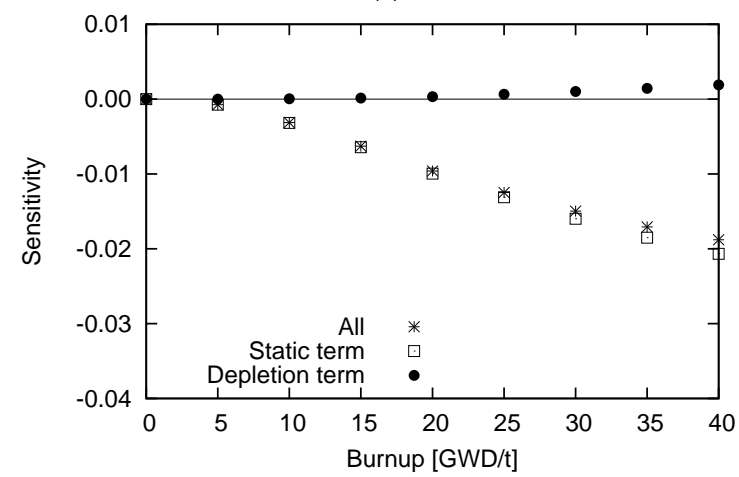

Figure 16 Energy-integrated sensitivity of $k_{\infty}$ after 5-year cooling with respect to capture cross sections of plutonium-241 and americium-241

G.Chiba

Uncertainty quantification of neutron multiplication factors of light water reactor fuels during depletion 


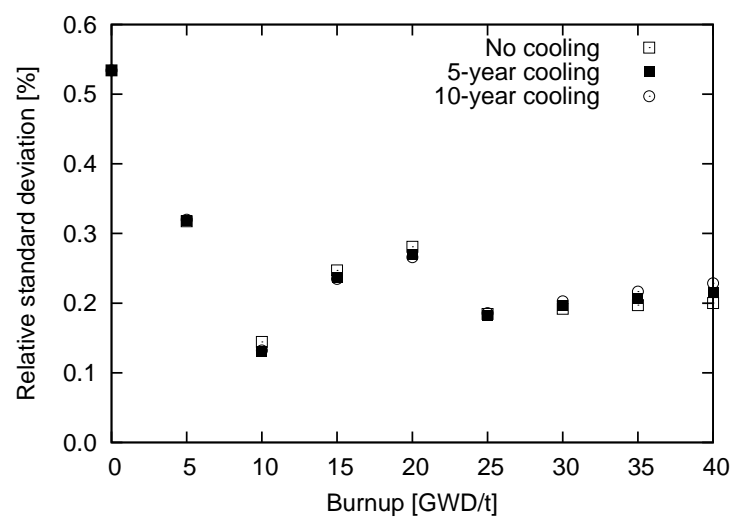

Figure 17 FP nuclides nuclear data-induced uncertainty of $k_{\infty}$ after specific cooling period

\section{G.Chiba}

Uncertainty quantification of neutron multiplication factors of light water reactor fuels during depletion 

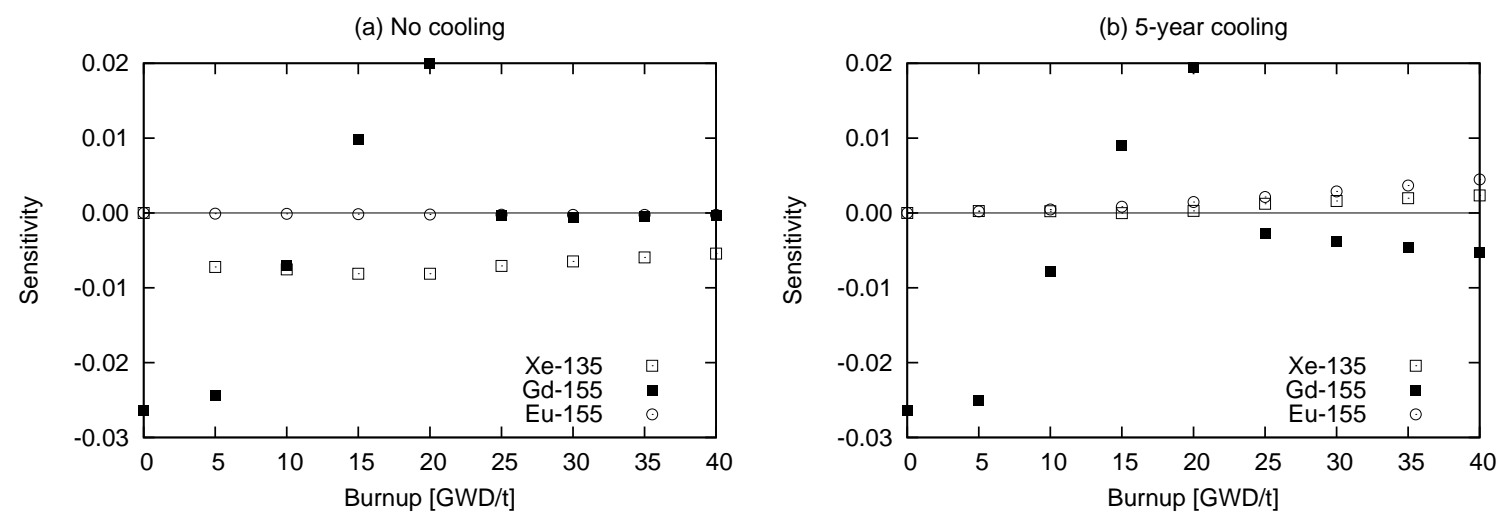

Figure 18 Energy-integrated sensitivity of $k_{\infty}$ in single cell with respect to capture cross sections of FP nuclides

G.Chiba

Uncertainty quantification of neutron multiplication factors of light water reactor fuels during depletion 